\title{
Enquête
}

Archives de la revue Enquête

5 | 1989

Biographie et cycle de vie

\section{Identité et cycle de vie}

Délimiter l'identité

Francesc Hernandez et Francesc Mercade

\section{(2) OpenEdition \\ Journals}

Édition électronique

URL : http://journals.openedition.org/enquete/101

DOI : 10.4000/enquete. 101

ISSN : 1953-809X

Éditeur :

Cercom, Éditions Parenthèses

Édition imprimée

Date de publication : 2 mars 1989

Référence électronique

Francesc Hernandez et Francesc Mercade, «Identité et cycle de vie », Enquête [En ligne], 5 | 1989, mis en ligne le 27 juin 2013, consulté le 19 avril 2019. URL : http://journals.openedition.org/enquete/101 ; DOI : 10.4000/enquete.101

Ce document a été généré automatiquement le 19 avril 2019. 


\title{
Identité et cycle de vie
}

\author{
Délimiter l'identité
}

\author{
Francesc Hernandez et Francesc Mercade
}

1 Tant du point de vue de l'individu que du point de vue de la division plus ample du social, le concept d'«identité » est un des moins transparents de ceux utilisés dans les écrits scientifiques. Les différentes lectures théoriques de la psychologie, de la sociologie, de l'anthropologie, de la psychologie sociale... peuplent les sciences sociales de définitions diverses et parfois contradictoires. En réalité il s'agit d'un thème extrêmement difficile à structurer. La plus grande partie des propositions prend pour base le concept du « moi abstrait » (le territoire et la population à partir du cadre communautaire), la référence suivante est la «caractéristique » de l'individu, la «forme » qu'il adopte au cours des étapes successives du développement (l'ethnie, la race, ou un moment culturel), le dernier degré est la définition de la personne comme « ensemble construit » d'attributs et de comportements (formations et structures sociales, accumulations culturelles). Du point de vue "structurel ", il faudrait inverser l'ordre des facteurs, pour mettre l'accent précisément sur le dernier point. Malgré la relation dialectique que les différents éléments entretiennent entre eux, il nous paraît évident qu'autant les individus que les collectivités sont le résultat d'un moment historique déterminé dans le développement culturel, politique et économique.

Quel sens cela a-t-il à l'aube du xxi siècle de réfléchir sur le concept d'identité ? Pour certains, les nouvelles technologies et la révolution cybernétique permettront une plus grande indépendance; pour d'autres, plus nombreux, les changements sociaux nous placent dans une situation de métropole universelle et homogène qui ne permet pas la plus petite nuance de différence, en dehors du monde des images et des représentations, de l'imaginaire et de l'irréel.

3 Les critiques du concept d'identité

4 Dans le domaine de la sociologie, nombreuses ont été les voix qui se sont élevées - directement ou indirectement - contre le concept d'identité. De Durkheim à Freud ou Marx, on a essayé - pour des raisons théoriques, méthodologiques et idéologiques diverses - de critiquer les concepts qui n'étaient pas facilement «objectivables » dans leur explication des phénomènes sociaux. 
Plus récemment, c'est Touraine qui a fait allusion au concept d'« identité » de manière très prudente ${ }^{1}$. Pour Touraine, l'identité sociale est le résultat d'un long processus d'intériorisation des valeurs qu'impose l'idéologie dominante. Les chemins de la socialisation montrent à l'acteur social ses possibilités d'adaptation à la société et les comportements et attitudes qui les rendent possibles.

6 L'approfondissement théorique et empirique du concept d'identité pourrait remplir - selon ces visions critiques - le rôle de la pensée sociale normative de l'Antiquité ou de la pensée chrétienne au Moyen Âge. La sociologie actuelle se convertirait en suivant ce chemin en une théologie moderne ou - comme Jacques Lacan désigne les sciences sociales - une technique orthopédique.

7 Au contraire, Touraine propose une critique des «illusions de l'identité », en étudiant la société dépouillée de ce premier masque. Les phénomènes sociaux ne se comprennent pas à partir des conduites rationnelles, les actions humaines se caractérisent par leur manque d'identité et même de conscience. L'identité d'une personne serait, de ce point de vue, le masque de sa soumission et de sa dépendance. En définitive, les sentiments qu'engendrent les processus d'identification renvoient surtout aux modèles sociaux qui sont imposés à l'individu de l'extérieur. Ils ne servent pas à définir la personne ellemême, mais à construire sa réalité depuis l'extérieur, engendrant un cadre aliénant.

L'ensemble des rôles sociaux qui sont proposés à l'individu, à travers la socialisation, repose sur un treillis complexe de relations sociales antagoniques qui essaient - par tous les moyens à leur disposition - de maintenir les conditions qui rendent possible l'inégalité. De cette manière, l'identité d'un individu - et d'un groupe - se rapporte fondamentalement au rôle qui lui a été attribué «socialement» et non pas à une définition autonome.

9 C'est ainsi que Touraine désigne les sociétés traditionnelles comme celles ayant produit une forte identité. Comme le signale Margaret $\mathrm{Mead}^{2}$, nous nous rapprochons d'un monde dominé par les cultures préfiguratives, dans lesquelles le poids de la tradition sera faible et où le regard se portera non sur le présent mais sur le futur. Certaines sociétés avancées se trouvent déjà à ce stade d'adaptation de leur propre futur. Notre société moderne définit l'identité en fonction de paramètres " prévus pour le futur » au lieu de chercher ses racines en regardant en arrière. Le concept d'identité perd de sa validité dans cette perspective.

Les difficultés d'une définition

11 Une première observation de notre vie quotidienne nous confronte à des comportements sociaux stéréotypés et à des modèles transmis par de puissants mécanismes à chaque citoyen. Cette "pénétration" dans l'individu construit un réseau complexe qui exerce une forte pression au cours des différents moments de sa vie: dans son travail, dans ses moments de loisirs, dans ses relations familiales... Depuis les situations de plus grande autonomie, privée ou intime, jusqu'aux relations sociales plus élargies, l'individu doit toujours se déplacer au milieu d'une invisible et complexe toile d'araignée qui se referme sur lui.

12 Il semble que la " société du futur » se fonde toujours sur le développement inexorable de la science et de la technique. D'un côté on prétend atteindre les niveaux les plus élevés de développement économique et de bien-être collectif, et de l'autre le pouvoir d'une élite pensante est en train de se consolider, en dominant tout le processus d'identification collective à l'aide des modèles culturels décrits antérieurement. 
13 Notre société hyper-développée, techno-industrielle, corporative, avancée, réussit une «unité sociale» nouvelle, basée - apparemment - sur la diversité, mais fondamentalement sur un processus de contrôle social à grande échelle qui entraîne des " adhésions inébranlables » ou des identifications passives qui ne sont pas conscientes du processus d'aliénation commencé et qui sont absorbées par la force d'une culture dite de masse et pour les masses, mais élaborée au fond de " tours d'ivoire ».

Bien que cela paraisse contradictoire, la pluralité apparente est précisément un des obstacles pour créer une identité collective critique et dynamique. Au sommet où se trouvent le regard sur soi (comment me vois-je moi-même?), la vision personnelle des autres (comment crois-je qu'ils me voient ?) et la réalité des lectures des autres (comment me voient-ils réellement ?), il existe un grand contraste d'optique.

Les définitions du concept d'identité doivent partir d'un modèle à « double entrée » : d'un côté il faut avoir la perspective individuelle (personnalité) qui concerne la structure organisée de l'identité personnelle, et de l'autre la vision sociale qui se rattache à la culture et au sentiment d'appartenance (nation) qui fait référence à la structure organisée de l'identité collective.

Identité, structures sociales et cycle de vie

À partir des réflexions antérieures, nous arrivons au noyau théorique qui nous intéresse dans le contexte de ce travail. Bien entendu, il est impossible, à partir de ces réflexions théoriques, d'épuiser l'analyse des processus d'identification collective. Le travail de recherche empirique - qualitativement et quantitativement - devrait compléter ces réflexions en cherchant de nouveaux indicateurs en relation avec le thème des structures sociales et des identités collectives. Malgré ces limitations, nous aimerions proposer une synthèse du cadre théorique dans lequel se situent les questions posées d'une manière qui dépasse la description pour aller vers l'analyse sociologique, dans le cadre d'un programme plus ample de recherche.

18 Nous avons déjà dit de quelle manière le concept d'identité se synthétise en plusieurs «moments » successifs qui forment la vie d'une collectivité. Les identités se construisent précisément à partir de relations sociales réifiées. L'identité collective est le résultat de la "somme » des différents cadres d'identification qui vont de l'individu au social. Les identités se fondent, donc, sur la permanence d'un ensemble de caractéristiques ou d'attributs qui sont exclusivement «propres». Les processus d'identification connotent une image d'inévitable (on ne peut pas «échapper » à sa propre identité). Rappelons les paroles de Baudelaire dans Les Fleurs du Mal et concrètement dans "L'Albatros » : «Exilé sur la terre au milieu des foules ses ailes de géant l'empêchent de marcher ».

19 Les déséquilibres au niveau des identités collectives sont précisément un des noyaux des mouvements nationalistes. L'incapacité d'intégrer diverses identités qui sont "propres » engendre une "pathologie sociale » qui condense une grande capacité de réaction. Les études de pathologie clinique des psychologues et des psychiatres - identité non intégrée, non-reconnaissance de soi-même dans le temps - sont en rapport avec ce que nous pourrions appeler les déséquilibres du sentiment collectif d'appartenance. Mais paradoxalement les études de l'identité dans une perspective individuelle ont déjà mis en relief le fait que chaque pathologie manque du renforcement par une composante sociale de l'identité. Le sentiment d'identité se consolide si on augmente les relations avec le monde « extérieur ». Dans des situations d'isolement (dont le type est Robinson Crusoe) la 
seule manière de survivre est de créer un monde extérieur imaginaire ; sans quoi on passe par une profonde crise personnelle.

De la même manière, du point de vue macro-sociologique, le sentiment d'identité est vu avec méfiance, l'analyse concerne surtout le versant " pathologique », mais on oublie que le sentiment d'identité devrait "additionner» les différents cadres d'appartenance (identités partagées). L'identité est précisément le chemin de la sociabilité. L'ensemble de mécanismes qui soutiennent l'identité d'un individu et sa projection sur les identités collectives est justement un élément fondamental de l'ordre social.

21 Prenant comme référence les voies par lesquelles un groupe social peut maintenir son identité, Sorokin les résume en quatre types : a) l'imposition d'un ensemble de lois et de règles qui laissent cependant une marge « d'interprétation personnelle »; b) la sélection des membres, en excluant ceux qui ne s'adaptent pas ; c) la transmission de modèles (idéologie) à ceux qui s'intègrent par la socialisation politique; $d$ ) la construction des mécanismes symboliques et rituels ${ }^{3}$. Cette approche repose implicitement sur une perception de l'identité qui implique l'existence d'attributs, invariables dans le temps. Mais il est important de souligner que le noyau de la continuité d'un groupe est justement l'identité et non les attributs. Ceux-ci peuvent être « différents ", dans le temps, tout en restant paradoxalement «invariables ", puisqu'ils continuent à être fidèles aux mêmes principes.

En prenant pour référence les identités nationales, beaucoup de mouvements politiques, et même certaines études apparemment "scientifiques », confondent l'identité avec les attributs. Un attribut déterminé peut même arriver à se substituer à l'identité. Confondre l'« invariabilité » des attributs avec le fait qu'ils ne peuvent pas réellement «changer ", empêche de comprendre le phénomène complexe des identités collectives. De fait, l'identité doit justement " changer » constamment, pour continuer à être "la même ». L'identité nationale, par exemple, subsiste mais se redéfinit constamment à travers l'histoire. Il nous apparaît évident que nous ne pouvons pas dire «l'identité nationale du Pays Basque est violente», ou «le nationalisme catalan est pacifique»; ces simplifications ne peuvent jamais être des affirmations sociologiques, car elles ne prennent pas en compte la dimension historique, qui est celle qui explique «la forme » des mouvements nationalistes à chaque moment culturel, social, politique et économique. En Espagne, les nationalistes nous offrent un champ de travail et de recherche exceptionnel pour l'étude des identités collectives et des cycles de vie. En continuant avec cet exemple, la formule des identités collectives partagées permet d'analyser les mouvements nationalistes dans le contexte de diverses couches de l'identité collective. Dans ce cadre d'analyse, il est compréhensible qu'une "même identité " permette différentes conduites, et même des conduites contradictoires, si elles sont analysées dans une perspective unique.

Les identités et leur expression dans les attitudes et la conduite humaine répondent à un principe « instrumental » (la capacité de se déplacer dans un espace social déterminé), mais répondent aussi à des éléments symboliques (la possibilité de partager et de transmettre des sentiments). Si nous imaginons, par exemple, un étranger vivant dans notre pays et qui maîtrise le français à la perfection, il est difficile qu'il arrive à dominer les jeux de mots, les phrases ingénieuses, les doubles sens, ou l'implication du ton et des inflexions de la voix. Dans ce cas-là, le rôle d'«étranger" a non seulement une signification fonctionnelle, mais aussi émotionnelle. 
24 L'identité est avant tout le lien conceptuel entre l'individu et sa culture. Dans l'ensemble des attributs qui définissent une nation, nous pouvons faire la distinction entre les «assignés» (territoire, langue, population), les «acquis» (caractère national, carte culturelle...), les « adoptés » qui répondent à un moment historique déterminé et qui ont un poids conjoncturel (sentiment de victime, utilisation de la violence, situation « pacifiste »...) et enfin, les éléments « supposés » que l'on peut distinguer des « adoptés » et qui peuvent être inclus dans le « caractère national » de manière essentialiste. Toutes ces catégories sont semblables à celles qui configurent l'identité d'une personne tout au long de son cycle de vie. Souvent l'analyse des identités collectives entend l'identité avec les sentiments dérivés de celles-ci. Quand il n'existe pas de conflits entre les différentes sphères d'appartenance, le sentiment d'identification reste au second plan et est moins conscient. Pour en revenir à l'exemple que nous avons utilisé plusieurs fois dans ce texte, quand il existe des conflits nationalistes, ce sentiment passe au premier plan et envahit tout le phénomène social qui, évidemment, est beaucoup plus ample et ne se réduit pas à ce seul aspect.

\section{NOTES}

1. A. Touraine, Introduccion a la sociologia, Barcelone, Ariel, 1978, p. 242-284.

2. M. Mead, Cultura y compromiso, Barcelone, Granica, 1977.

3. P. A. Sorokin, Sociedad, cultura y personalidad, Madrid, Aguilar, 1973. 